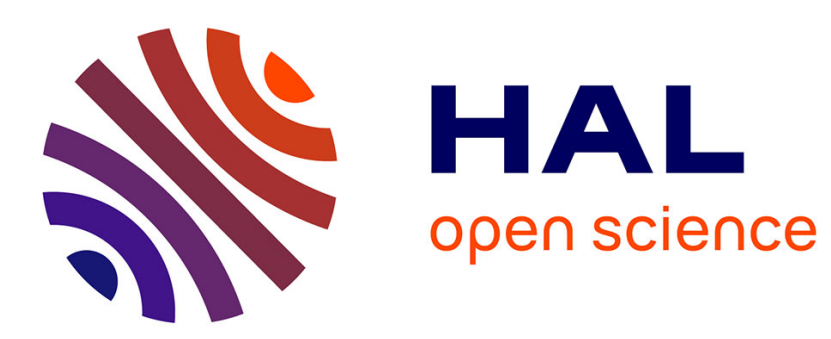

\title{
Pore scale modelling of DNAPL migration in a water-saturated porous medium
}

Khalifa Nsir, Gerhard Schäfer, Raphaël Di Chiararoupert, Lionel Mercury

\section{To cite this version:}

Khalifa Nsir, Gerhard Schäfer, Raphaël Di Chiararoupert, Lionel Mercury. Pore scale modelling of DNAPL migration in a water-saturated porous medium. Journal of Contaminant Hydrology, 2018, 215, pp.39-50. 10.1016/j.jconhyd.2018.07.001 . insu-01856217

\section{HAL Id: insu-01856217 https://hal-insu.archives-ouvertes.fr/insu-01856217}

Submitted on 10 Aug 2018

HAL is a multi-disciplinary open access archive for the deposit and dissemination of scientific research documents, whether they are published or not. The documents may come from teaching and research institutions in France or abroad, or from public or private research centers.
L'archive ouverte pluridisciplinaire HAL, est destinée au dépôt et à la diffusion de documents scientifiques de niveau recherche, publiés ou non, émanant des établissements d'enseignement et de recherche français ou étrangers, des laboratoires publics ou privés. 
1 Pore scale modelling of DNAPL migration in a water-saturated

\section{porous medium}

a Laboratoire d'Hydrologie et de Géochimie de Strasbourg (LHyGeS), UMR 7517, Université de Strasbourg/EOST, Centre National de la Recherche Scientifique (CNRS), 1 rue Blessig, 67084 Strasbourg, France.

Corresponding author: Gerhard Schäfer, schafer@unistra.fr

\section{Abstract}

A numerical simulator based on the discrete network model approach has been developed to simulate drainage processes in a water-saturated porous medium. To verify the predictive potential of the approach to simulate the unstable migration of adense nonaqueousphase liquid (DNAPL) at the pore scale, the numerical model was applied to laboratory experiments conducted on a sand-filled column. The parameters relative to pore body size and pore throat size used in the construction of the equivalent network were derived from discrete grain-size distribution of the real porous medium. The observed water retention curve (WRC) was first simulated by desaturation of the network model.The good agreement of the modelled WRC with the experimental one highlights that the applied approach reproduces the main characteristics of the real pore space. The numerical model was then applied to rate controlled experiments performed on a homogenous sand-filled column to study the gravity-driven fingering phenomenon of immiscible two-phase flow of water and a DNAPL. The numerical results match within $10 \%$ based on the standard deviation with the experiments. They correctly 
downward flow) and the flow rate, on the stability of the water/DNAPL front in a saturated porous medium.

Keywords: network model; porous medium; WRC; DNAPL; immiscible displacement; fingering

\section{Introduction}

Characterization and quantification of the migration of immiscible liquids in groundwater are topics that have received considerable attention in recent years (Alexandra et al.2012; Wu et al.2017). Several studies of two-phase immiscible flows have been motivated by the need to treat spills and leaks of so-called dense nonaqueousphase liquid (DNAPL), which can severely impact the quality of subsurface water supplies (Kueper and Frind 1989; Birovljev et al. 1991; Fayers et al. 1996; Bettahar et al. 1999). When infiltrating throughthe saturated zone, migration of DNAPLcan result in highly fingered fluid distributions. The occurrence of fingering is caused by flow instabilities due to differences in viscosity and density between DNAPL and water (Khataniar and Peters 1992; Riaz and Tchelepia 2006; Essaid et al. 2015; Cheng et al. 2016). These fingers propagate rapidly, causing early breakthrough relative to stable displacement (Brailovskyet al. 2006).

Numerical modelling of DNAPL displacement in water-saturated porous media using continuum models has been performed by many researchers (Nayagum et al. 2004; Aggelopoulos and Tsakiroglou 2009, Zhang et al. 2011; Erning 2012; Kokkinaki et al. 2013; Sleep et al. 2015, Schneider et al. 2015). However, the fingering process in a homogenous porous medium cannot be simulated with these models because they do not explicitly consider the instability behaviourof the DNAPL-water interface.

To overcome this shortcoming, microscopic approaches such as Stokes' solution for flow in single pores with a specified geometry or lattice Boltzmann methods(LBM) have been 
51 investigated to reproduce unstable displacement experiments. Kiriakidis et al. (1991) developed 52 a 2D algorithm involving Monte Carlo decision-making, random walks, and principles of percolation theory. This algorithm successfully predicted the three distinct behaviours of immiscible displacement in porous media, such as viscous fingering, stable displacement, and invasion percolation, but the model did not include the gravity term, and gravity fingering was not considered. Ewing and Berkowitz (1998) developed a generalized growth model based on invasion percolation to simulate immiscible displacement in saturated porous media. Even capillary, viscous and gravity forces were incorporated in their 3D algorithm, where viscous forces were expressed stochastically rather than explicitly. Glass et al. (2001) developed a macro-modified invasion percolation (MMIP) model, including also the effects of all 3 forces within the invading phase in a macro-heterogeneous porous medium. The simulated evolving invasion structure during the gravity-destabilized condition matched the experimental distributions quite well.Dong et al.(2011) proposed a LBM model taking into account both wettability and gravity tosuccessfully reproduce flow experiments in Hele Shaw cells.Recently, Liu et al.(2013; 2014; 2015) have developed a Lattice Boltzmann model that was able to simulate the main flow regimes (capillary fingering, viscous fingering and stable displacement). The model was applied to simulate drainage micromodel experiments of the liquid $\mathrm{CO}_{2}$ displacement of water.The authors obtained a good agreement with the experimental measurements and showed that increasing $M$ (viscosity ratio of non-wetting fluid to wetting fluid) or decreasing the media heterogeneity can enhance the stability of the displacement 71 process.

While the lattice Boltzmann method gives a more rigorous description of different physical processes, it is often limited to relatively simple physical situations or to systems encompassing only a few pores. For this reason, pore-scale networks are often used to describe 
the flow rate of fluids at the pore scale (Koplick and Lasseter 1985; Aker et al. 1998; Helge et al. 1999; Jia et al. 1999; Laroche and Visika 2005).

In pore-scale network modelling, local capillary equilibrium via the Young-Laplace equation is generally used to determine multiphase fluid distribution. A set of rules that describes the appropriate physical processes and arrangements of fluid within each pore is developed and then combined to describe the displacement of both fluids in the system. Macroscopic behaviours of the displacement can then be seen in network models, even though they are not coded explicitly.

Many network models have been developed to study a wide range of displacement processes, including drainage and imbibition (Nordhaug et al. 2003; Singh and Mohenty 2003; Ferer et al. 2007; Niaser et al. 2009; Tørå et al. 2012; Sheng and Thompson 2013; Bultreys et al. 2015; Kallel et al. 2017). Regular lattice structures are generally used, with pore bodies corresponding to the vertices of the lattice and pore throats connecting the pore bodies. The most important aspects of network models are the dimension and coordination (Gaoet al. 2012; Jivkovet al. 2013). For example, trapping is a dominant process in 2D, less important in 3D, and virtually non-existent in 4D (3D+time) and when an above multi-directional pore-network is used (Raoof and Hassanizadeh 2010). Likewise, networks with high coordination behave quite differently than networks with low coordination. In most early network models, pore space parameters were chosen without reference to a realistic porous medium. Network is equivalent only in a statistical sense to the modelled system. Indeed, they were used to explore network concepts as much as to explore porous media. More modern network models were often built directly from micro-CT scans of the rock, and so they had not only the same pore size distribution but also the same exact structure as the rock they are simulating (Al-Raoush and Willson 2005; Prodanovic et al. 2007; Dong and Blunt 2009; Raoof et al. 2010; Bultreys et al. 2015). In these cases, the ability to reproduce physical measurements is excellent. However, 
100 if the exact rock type has to be used for the network construction, the application of predictive

101 pore-scale modelling will be severely limited due to the complexity and cost of the methods.

102 More details about the methods for obtaining pore space information, constructing pore

103 networks and the application of pore network models in porous media can be found elsewhere

104 (see Joekar-Niasar and Hassanizadeh(2012) and Xionget al. (2016)).

105 By controlling parameters such as pore-size disorder, viscosity, and flow rate, previously

106 developed dynamic pore network models were used successfully to reproduceviscous fingering

107 phenomena exhibited in laboratory studies.Lenormandet al. (1988) developed a dynamic

108 drainage pore network model and used it to simulate corresponding micromodel experiments.

109 Their model reproduces capillary fingering, viscous fingering and frontal displacement regimes

110 for different capillary number and mobility ratio combinations successfully. The largest

111 network that they used in their simulations consisted of $100 \times 100$ nodal junctions and they

112 presented a relatively limited set of simulation results. Aker et al. (1998) developed a two-

113 dimensional network simulator and used it to characterize the different flow regimes observed

114 in drainage. They introduced a new method that allowed the simultaneous flow of two liquids

115 into one pore and demonstrated that viscous fingering could be reproduced. However, the

116 approach was highly CPU intensive and a $60 \times 80$ network was the largest lattice that could be

117 considered. In addition, they only presented results before a breakthrough of the injected

118 phase.Singh and Mohanty (2003) developed a dynamic network model providing 3D results

119 consistent with the 2D results ofLenormandet al.(1988). Their simulator could reproduce the

120 displacement front structure and dynamics as function of capillary number and viscosity ratio.

121 Whilst their simulator could produce various displacement regimes, including viscous

122 fingering, the largest system used in their study was a $30 \times 8 \times 8$ network. Tørå et al. (2012)

123 extended the model developed by Aker et al. (1998) by incorporating the dynamics of the

124 wetting layers using an approach similar to Singh and Mohanty (2003). They used this model 
to study saturation profiles during imbibition and the resistivity index at different capillary numbers. Their numerical results were in good agreement with experimental data from sandpacks. The simulations were carried out on one realization of model volume of $(4.5 \times 1.5 \times 1.5) \mathrm{mm}^{3}$ consisting of 767 nodes and 1750 tubes.

Most of the previous pore network modelling studies described above were performed in

130 small networks and were followed by a successful benchmarking exercise of the numerical

131 simulations against micromodel experiments. However, the modelling approach is rarely used

132 to simulate such mechanisms at the laboratory core scale. Additionally, very few studies have

133 presented saturation maps after breakthrough under unstable conditions. An integrated effort is

134 needed to connect network models and laboratory tests at the core scale more closely.

135 The main objective of the present work is to test, at the scale of a few centimetres, the

136 capacity of the pore scale network model developed by Nsir and Schäfer (2010) to capture the

137 gravity-driven fingering observed in our own water-DNAPL drainage system. The model is

138 first applied to predict the water retention curve of the model sand used in the experiment. The

139 comparison of the experimental curve and the one modelled will show whether the used

140 network model reproduces the main characteristics of the real pore space. In a second step, the

141 numerical model is then used to simulate the displacement of water by DNAPL during

142 controlled drainage experiments performed in a $68-\mathrm{cm}$ long glass column filled with the

143 homogenous model sand. The numerical results are compared to the observed physical

144 quantities, such as the distribution of arrival times of the DNAPL/water front at a control

145 section, the DNAPL pressure at the inlet as a function of time, and the DNAPL saturation profile

146 at the end of the displacement experiment. 

diameter of $10 \mathrm{~cm}$. The used porous medium is a medium-sized quartz sand with a mean grainsize diameter of $0.4 \mathrm{~mm}$, a uniformity coefficient of 2.1 and a low fraction of organic content (foc $=0.09 \%$, based on NFT 31-109) (Bohyet al. 2006). The hydraulic properties of the quartz sand, such as its moisture characteristics,were quantified in former studies by Cotel(2008).

Trichloroethylene (TCE) was chosen as the DNAPL for the experiments because it is among

155 the most frequently detected contaminants in subsurface environments. At $20^{\circ} \mathrm{C}$, TCE has a density of $1.463 \mathrm{~g} / \mathrm{cm} 3$, a viscosity of $0.0056 \mathrm{~g} / \mathrm{s} / \mathrm{cm}$, and a solubility in water of $1300 \mathrm{mg} / \mathrm{L}$. of TCE in an initially water-saturated homogeneous porous medium. The outlet section of the column was kept at a constant water pressure. A highaccuracy peristaltic pump with flow rates

160 up to $200 \mathrm{~mL} / \mathrm{min}$ was used to inject the displacing fluid in the sand-filled column. Pressures at the inlet and outlet were measured using a pressure transducer (Cerabar T-PMC131). Several system parameters (e.g., flow rate, flow mode (upward flow, downward flow) were varied in 163 the experimental programme.

The experimental approach is original, as it is based on the use of optical fibres to locally quantify the arrival times of the DNAPL/water front at different points within a control section of the porous medium. Local DNAPL saturations at different depths of the sand column were also quantified by in situ soil sampling. Because of the emplacement of fibres, the sampling was achieved only above the sensor control section of the column (between $z=0$ and $z=-34$ $\mathrm{cm})$. At each sampling depth, one sample was taken on the axis of the column and two others

170 on opposite points of the edges of the column section. More details about the experimental setup 171 and sampling method are given in Nsiret al. (2012).

\section{Numerical model}




\subsection{Geometry of the network}

A 3D network model based on spherical porebodies and cylindrical porethroats was developed to simulate drainage processes in a saturated porous medium. The pore space is represented by a cubic lattice with porebodies corresponding to the vertices of the lattice and

178 porethroats connecting the porebodies. The connectivity or coordination number in the network

179 (the number of throats connected to a given pore in the network) is set equal to 6 . The pore

180 body and pore throat radii were drawn at random using a probability frequency distribution

181 given by a special case of the Weibull distribution (Weibull, 1951). We used this type of

182 distribution because it is easy to implement, and its model parameters may adequately represent

183 a given pore size distribution. Compared to other models, such as normal, lognormal or bimodal

184 distribution, the probability of finding smaller pore bodies in the network is higher. In the

185 modelling approach, specified values of the minimum, mean and maximum radii $\left(\mathrm{r}_{\text {min }}, \mathrm{r}_{\text {mean }}\right.$,

186 and $r_{\max }$, respectively) are needed to achieve the pore size distribution. The probability

187 distribution used is as follows:

$g(x)= \begin{cases}\frac{x}{x_{2}^{2}} \exp \left(-\frac{x^{2}}{x_{2}^{2}}\right) & \text { for } x \leq x_{3} \\ 0 & \text { for } x \succ x_{3}\end{cases}$

189 where the radius is $\mathrm{r}, \mathrm{x}=\mathrm{r}-\mathrm{r}_{\min }, \mathrm{x}_{2}=\mathrm{r}_{\mathrm{mean}}-\mathrm{r}_{\text {min }}$, and $\mathrm{x}_{3}=\mathrm{r}_{\max }-\mathrm{r}_{\min }$. Both porebody and

190 porethroat radius distributions are of this type, although with different parameters: $r_{\text {min }}, r_{\text {mean }}$

191 and $\mathrm{r}_{\max }$. For a throat connecting pores $i$ and $j$, the throat length is defined as $\mathrm{L}_{\mathrm{ij}}=\mathrm{L}-\left(\mathrm{r}_{\mathrm{pore}, \mathrm{i}}+\right.$

$192 \mathrm{r}_{\text {pore, }}$ ), where Lis the distance between the centres of two adjacent pore bodies. $\mathrm{L}$ is considered

193 constant in the chosen network. The values of $r_{\min }, r_{\text {mean }}$, and $r_{\max }$ specified for both pore body

194 and pore throat and thus for the node spacing $\mathrm{L}$ are hereafter considered as the geometric

195 parameters of the network model.

196 3.2. Governing flow equations 
When solving the transient flow field, the network model takes into account capillary variations in space, the local viscous pressure field, and gravity. The location of fluid-fluid interfaces is restricted to the connection between the pore body and pore throat. The Washburn equation (Washburn, 1921) is used to quantify the flow rate in a pore throat, which is connected to a pore body separated by a water-DNAPL interface. The main assumptions are as follows:

202 (i) fluids used are Newtonian, incompressible, and immiscible; (ii) flow is laminar; and (iii) 203 local capillary pressure in a pore bodyis negligible, resulting in only one mean pressure within a pore body independent of the phase saturation of that pore body. The mass balance equation for each fluid phase $\alpha$, wetting (water, $\alpha=w$ ) and non-wetting (DNAPL or air, $\alpha=n w$ ), in a pore 206 bodyiisas follows:

$\mathrm{V}_{\mathrm{i}} \frac{\partial \mathrm{S}_{\mathrm{i}}^{\alpha}}{\partial \mathrm{t}}+\sum_{\mathrm{j}} \mathrm{Q}_{\mathrm{ij}}^{\alpha}=\mathrm{Q}_{\mathrm{i}}^{\alpha, \text { inlet }}$

where $\mathrm{V}_{\mathrm{i}}\left[\mathrm{L}^{3}\right]$ represents the volume of pore bodyi, $\mathrm{S}_{\mathrm{i}}{ }^{\alpha}[-]$ represents local saturation (percent of $V_{i}$ filled with fluid $\left.\alpha\right), Q_{i j}{ }^{\alpha}\left[L^{3} \mathrm{~T}^{-1}\right]$ is the phase flow rate through a pore throat connecting pore bodyiand pore body $\mathrm{j} . \mathrm{Qi}^{\mathrm{a}}{ }^{\text {,inlet }}\left[\mathrm{L}^{3} \mathrm{~T}^{-1}\right]$ is the external phase flow rate into pore $\mathrm{i}$, which is zero,

211 unless the pore is located at the flow boundary. The sum runs over all neighbouring pores of 212 pore bodyi.

213 Summation of Eq. (2) over the two phases gives the following equation for each pore 214 body i:

$$
\sum_{j}\left(Q_{i j}^{w}+Q_{i j}^{n w}\right)=\sum_{j} Q_{i j}=Q_{i}^{n w, i n l e t}
$$

The expression used for the phase flow rate $\mathrm{Q}_{\mathrm{ij}}$, depends on the fluid present in the pore

217 throat connecting pores $\mathrm{i}$ and $\mathrm{j}$, and on the presence of a meniscus at the boundaries between a 218 pore body and a pore throat.

If no meniscus is present at the pore throat entry, the flow rate $\mathrm{Q}_{\mathrm{ij}}$ through the pore throat 220 is given by Poiseuille'slaw, as follows: 
$221 Q_{i j}=\frac{\pi r_{i j}^{4}}{8 L_{i j} \mu_{\alpha}}\left(P_{i}-P_{j}-\left(z_{i}-z_{j}\right) \rho_{\alpha} g\right)$

222 where $\mu_{\alpha}\left[\mathrm{ML}^{-1} \mathrm{~T}^{-1}\right]$ and $\rho_{\alpha}\left[\mathrm{ML}^{-3}\right]$ are thedynamic viscosity and the density of the phase fluid 223 present in the pore throat, respectively; $\mathrm{r}_{\mathrm{ij}}$ and $\mathrm{L}_{\mathrm{ij}}$ are the radius and the length of the pore throat, 224 respectively; $P_{i}$ and $P_{j}$ are the fluid pressures in pores $i$ and $j$; and $z_{i}$ and $z_{j}$ are the height of the 225 vertices at the centre of pore bodiesi and $\mathrm{j}$. The first term of the right-hand side of Eq. (4), $226 \pi \mathrm{r}_{\mathrm{ij}}{ }^{4} /\left(8 \mathrm{~L}_{\mathrm{ij}} \mu_{\alpha}\right)$, represents the flow conductance in the pore throat.

227 If a meniscus is present at the pore throat entry, the flow rate Qij through the pore throat 228 is expressed by the Washburn equation (Washburn, 1921), as follows:

$$
Q_{i j}=H\left\langle P_{i}-P_{j}-\left(z_{i}-z_{j}\right) \bar{\rho} g-P_{c}\left(r_{i j}\right)\right\rangle \frac{\pi r_{i j}^{4}}{8 L_{i j} \bar{\mu}}\left(P_{i}-P_{j}-\left(z_{i}-z_{j}\right) \overline{\rho g}\right)
$$

230 where $\mathrm{P}_{\mathrm{c}}\left(\mathrm{r}_{\mathrm{ij}}\right)\left[\mathrm{ML}^{-1} \mathrm{~T}^{-2}\right]$ denotes the threshold capillary pressure that must be exceeded for the 231 DNAPL to enter the pore throat connecting pore bodiesi and $\mathrm{j}$. This threshold pressure is given 232 by the Young-Laplace equation, as follows:

$$
P_{c}\left(r_{i j}\right)=\frac{2 \sigma \cos \theta}{r_{i j}}
$$

234 where $\theta$ is the contact angle (assumed to be zero in this work). $\mathrm{H}<\mathrm{X}>$ is a Heaviside function that stands for the displacement condition of the interface in a pore throat. The throat can be 236 blocked by capillarity if the capillary entry pressure $\left(\mathrm{P}_{c}\left(\mathrm{r}_{\mathrm{ij}}\right)\right)$ has not yet been reached. The 237 Heaviside function is defined as follows:

$238 \mathrm{H}\langle\mathrm{X}\rangle= \begin{cases}1 & \text { for } \mathrm{X} \succ 0 \\ 0 & \text { for } \mathrm{X} \leq 0\end{cases}$

239 If a meniscus is present at the boundary between a pore body and a pore throat, the identity 240 of the fluid flowing through the pore throat (DNAPL or water, or capillary-blocked) can only 241 be determined after computation of the pressure field in the network. Therefore, an effective 242 viscosity and an effective density are used for the displacing fluid in Eq. (5) and are defined by 
the following:

$$
\begin{aligned}
& \bar{\mu}=\mu_{n w} S_{i j}^{n w}+\left(1-S_{i j}^{n w}\right) \mu_{w} \\
& \bar{\rho}=\rho_{n w} S_{i j}^{n w}+\left(1-S_{i j}^{n w}\right) \rho_{i j}^{w} \\
& S_{i j}^{n w}=\left(S_{i}^{n w}+S_{j}^{n w}\right) / 2
\end{aligned}
$$

245 where $\mathrm{S}_{\mathrm{ij}}{ }^{\mathrm{nw}}[-]$ is the average phase saturation between pore bodiesi and $\mathrm{j}$. As the average phase 246 saturation evolves as a function of time, it indirectly accounts for the interface movement inside 247 a pore throat.

248 Introducing the appropriate expression of flow rate $Q_{i j}$ in Eq. (3) leads to a set of algebraic 249 equations with the pore body pressures as unknowns. This set of equations is written in matrix 250 form, as follows:

$251 \quad \mathrm{~A} \times \mathrm{B}=\mathrm{P}$

252 where $\mathrm{A}$ is the conductance matrix with elements that depend on the connections between 253 different throats, Pdenotes the pressure vector, and the Bvector contains the pressure and the 254 flow rate at the boundaries, the buoyancy terms, and the capillary pressure if a meniscus is 255 present in the pore throat.

\subsection{Numerical solution procedure}

The governing equations presented in the previous section are solved for fluid pressure $\mathrm{P}$ and wetting fluid saturation $\mathrm{S}_{\mathrm{w}}$ using an IMPES (IMplicit Pressure-Explicit Saturation) modelling approach. The flow chart describing the different steps of the pore-scale network model is shown in Figure 1.

Equation (7) is solved implicitly for $\mathrm{P}$, assuming that $\mathrm{S}_{\mathrm{w}}$ and throat conductances $\pi \mathrm{r}_{\mathrm{ij}}{ }^{4} /(8$ $\left.\mathrm{L}_{\mathrm{ij}} \mu_{\alpha}\right)$ are known from the previous time step. The calculated pressure field is then used to quantify fluid flow rates through the pore throats. The ratio of free volume in a pore body $\mathrm{i}$ (not

264 invaded by displacing fluid) to the sum of flow rates in all pore throats connected to pore body $265 \mathrm{i}$ and containing a moving interface gives the filling time needed for that pore body $\mathrm{i}$ to be 
invaded. The minimum value of the calculated filling times (hereafter called $\mathrm{T}_{\min }$ ) is then used

to update the local saturation in each pore body as follows:

268

$$
\mathrm{S}_{\mathrm{i}}^{\mathrm{t}+1}=\mathrm{S}_{\mathrm{i}}^{\mathrm{t}}+\frac{\mathrm{Q}_{\mathrm{i}} \times \mathrm{T}_{\text {min }}}{\mathrm{V}_{\mathrm{i}}}
$$

where $\mathrm{Si}_{\mathrm{i}}^{\mathrm{t}}[-]$ and $\mathrm{Si}_{\mathrm{i}}^{\mathrm{t}+1}[-]$ denote the saturation at the previous time $(\mathrm{t})$ and calculated at the new 270

time $(\mathrm{t}+1)$, respectively; and $\mathrm{Q}_{i}\left[\mathrm{~L}^{3} \mathrm{~T}^{-1}\right]$ represents the sum of flow rates in all pore throats connected to pore body $i$ and containing a moving interface.

During one time step, only one pore body reaches the full non-wetting phase saturation. This pore body generates additional interfaces at all connecting pore throats that are filled with the wetting fluid. Newly created interfaces are tested for stability, and appropriate expressions for flow rate $\mathrm{Q}_{\mathrm{ij}}$ are then identified. This modifies the conductance matrix at each time step. The field pressure is computed, and the procedure for updating the saturation in pore bodies is repeated. This provides a transient response for the pressure and saturation fields.

To solve the set of equations (9), a Preconditioned Conjugate Gradient Method (PCGM) algorithm is used. The simulations are conducted on a Linux-computer with 3956 MB RAM and a Celeron processor running at $1998 \mathrm{MHz}$. One iteration on the chosen lattice network $(20 \times 20 \times 200)$ took approximately 90 seconds and required a memory space of approximately 4 MB. The corresponding Central Processing Unit (CPU) time was approximately twenty days for the chosen lattice size. As the Fortran code was not parallelised and the free memory offered by the used computer was limited, simulations could not be launched when a lattice size larger than $20 \times 20 \times 200$ was used.

The time-stepping process is repeated until a stopping condition is reached. This condition allows the choosing of the stage at which the simulation should be achieved: after attaining a fixed saturation value at a given cross-section, after reaching a breakthrough of the 
invading fluid phase, after having performed a defined number of steps, or after the injecting 290 of a predefined total volume of the invading fluid phase.

\subsection{Model parameters and numerical implementation}

To obtain a reliable simulation of laboratory experiments, the characteristic parameters of size distributions for both pore bodies and pore throats $\left(r_{\min }, r_{\text {mean }}\right.$ and $\left.r_{\max }\right)$ werecomputed 294 based on the grain-size distribution (GSD) of the real porous medium. Themodelling concept is 295 based on the geometric properties of packing spheres (Dodds, 1980), which are combined with a probability approach proposed by Rouault and Assouline (1998). The input data for sphere packing are in the form of a discrete particle-size distribution derived from the (GSD) of the sand used in the experiment (Nsir and Schäfer, 2010). The obtained results consist of one pore body-sized distribution curve and four pore throat-sized distribution curves. Assuming that the generated pore body sizes and pore throat sizes are normally distributed, which is generally assumed for a large sampling number, assessment of the mean value and the standard deviation (sd) of corresponding curves allows the computation of various ranges of pore body radii and pore throat radii in terms of $r_{\text {min }}, r_{\text {mean }}$ and $r_{\text {max }}$. More details of the applied method are given in 304 the work of Nsir and Schäfer (2010).

The geometric parameters for the pore body radii and pore throat radii considered in the numerical simulations are shown in Table 1. The grid length should be slightly two times greater than the maximum radius of pores $\left(\mathrm{r}_{\max }=0.054 \mathrm{~mm}\right)$. For this reason, the distance between the centres of two adjacent pore bodies (L) was fixed to $0.14 \mathrm{~mm}$. The chosen values gave the best

309 fit for the measured macroscopic properties of the medium sand used in the experiment. The 310 equivalent intrinsic permeability and porosity are $8.6 \times 10^{-11} \mathrm{~m}^{2}$ and $47 \%$, versus the measured 311 values of $9 \times 10^{-11} \mathrm{~m}^{2}$ and $40 \%$, respectively (Nsir and Schäfer, 2010). By fixing the maximum 312 radii $\left(\mathrm{r}_{\max }\right)$ and the minimum radii $\left(\mathrm{r}_{\min }\right)$ of the size distribution as one time, two times and three 
times the standard deviation (sd)away the mean radius ( $r_{\text {mean }}$ ), threesize distributions with

314 different shapeswere considered for a given lattice size (see Table 1).

To simulate the entire laboratory column $(68 \mathrm{~cm}$ long, $10 \mathrm{~cm}$ internal diameter), an equivalent network with $714 \times 714 \times 4858$ nodes in the $[\mathrm{X}, \mathrm{Y}, \mathrm{Z}]$ directions, corresponding to $2,476,588,968$ pore elements is required. Simulations with such lattice size are highly CPU

318 intensive and RAM consuming and were, consequently, not manageable by our computational

319 resources. To face this difficulty, we decided to reduce the size of the network in order to match 320 the maximum height and width of the column.

An equivalent network of $7 \times 7 \times 4858$ pore bodies was initially chosen to simulate drainage

322 experiments. This spatial discretisation has the advantage of reproducing the total height of the 323 physical model and, consequently, gravity effects, even when only a small fraction of the 324 column section is used ( 1 square millimetre). This might favour the formation of very few 325 fingers that might occupy most of the cross-section of the model. As they move downward, the 326 number of pore bodies to invade before reaching the bottom might increase the drainage of the 327 porous medium. Consequently, an overestimation of the saturation of the invading fluid can be 328 induced by a coarse pore discretisation in the transverse direction. Using the correct shape of 329 the cross-section is thus as important as the real column height.

To account for a sufficiently large section of the medium that is manageable by our proper 331 computational resources, additional simulations considering networks with more nodes along 332 the two directions of the horizontal section $(20 \times 20$, rather than $7 \times 7)$ but with minimum nodes 333 in the direction of flow (200, instead of 4858) have also been undertaken. To conserve 334 representation of the total physical height, the node-spacing was increased $(3.4 \mathrm{~mm}$, instead of $3350.14 \mathrm{~mm}$ ). Increasing the node spacing does not impact the macroscopic properties of the 336 equivalent network as already discussed in the previous work of Nsirand Schäfer (2010). 337 Furthermore, the corresponding network may be assimilated in this case to a package of 
cylindrical tubes joining at junctions with volume, which remains the physical representative of reality.

To reproduce the conditions of the performed drainage experiment, the network model

341 was initially water-saturated and a constant flow rate of $29.6 \times 10^{-4} \mathrm{~mL} / \mathrm{min}$ of DNAPL was 342 applied at the inlet section of the model. As the simulated cross-section of the network was 343 significantly smaller than the real cross-section of the experimental soil column, the simulated 344 injection rate was reduced to the experimental injection rate by the ratio of the cross-sectional area of the model to that of the column. At the outlet section of the model, the observed water head was specified as a constant pressure boundary. The simulation was stopped when the time needed to inject the DNAPL volume considered in the experiment was reached.

\section{Results and discussion}

\subsection{Water retention curve modelling}

The relationship between the amount of water, typically quantified by the degree of saturation (S) or volumetric water content $(\theta)$, and pore-water potential, typically quantified by matric potential or capillary pressure $\left(\mathrm{P}_{\mathrm{c}}\right)$, is referred to as the water retention curve $(\mathrm{WRC})$ of soils. A key attribute of the developed model is its capability to compute fluid pore-scale saturation as a function of capillary pressure applied to a given lattice, which allowsmodelling the WRC of a porous medium.Comparison of the numerically calculated WRC with the experimentally obtained oneallowsfor verificationif thepredicted pore space parameters match the features of WRC observed ina column experiment.

The experimental data used for verification were obtained independently in a multistep 360 outflow experiment (Cotel, 2008). To reproduce the functional relationship between capillary 361 pressure and water saturation, a displacement simulation was performed using a series of sequential capillary pressure steps. The water saturation of the porous medium was computed 
at the end of each displacement step. For each prescribed capillary pressure, the dynamics of

364 the desaturation of the medium is quantified by the track of the generated interfaces in the pore network. This rule was applied iteratively until all the air/water interfaces remainedat rest under

366 the given capillary pressure level, andthe invasion of water-filled pores by air is stopped. At 367 this stage, the whole network is under hydrostatic conditionsand the corresponding water 368 saturation can thus becomputed. Then, the applied capillary pressure is again increased. The 369 simulation is stopped when the air/water front reached the outlet face of the network.

The simulations were conducted on a network of $20 \times 20 \times 200$ nodes because it has lower

371 CPU consumption and presents a larger cross section than the $7 \times 7 \times 4858$ lattice. Furthermore, 372 matching a fine vertical discretisation of the column is not absolutely necessary to model the 373 macroscopic capillary pressure as a function of the water saturation. According to the 374 experimental data, the first capillary pressure applied to the network boundary was fixed to 100 375 Pa. The network was then drained stepwise by systematically incrementing the prescribed 376 capillary pressure by a value of $100 \mathrm{~Pa}$ at each displacement step.

The simulated WRC for different pore throat size distributions compared to the measured 378 one are given in Figure 2. The agreement in the sloping of the curves shows saturation changes 379 in a similar fashion for a given change in pressure. The model seems to be able to adequately 380 predict the observed capillary pressure-saturation relationship up to a capillary pressure of 381 approximately $0.2 \times 10^{+4} \mathrm{~Pa}$ (equivalent to $0.2 \mathrm{~m}$ water head). The model predicts slightly higher 382 water saturations than those measured in the experiment. However, it clearly deviates from the 383 experimental data set for high capillary pressures corresponding to water saturations lower than 3840.2 . The mobilisation of the wetting phase in the simulated network appears significantly higher 385 than that observed in the experiment in this condition. This may be due to limitations of the 386 numerical model when a network section of only $(20 \times 20)$ pores in a horizontal transect is used. 387 In fact, neglecting the spatial pore size correlations or connectivity may lead to inaccurate 
predictions of water retention at very low water saturation. In addition, the simulated fluid

389 displacement is considered frontal. Thus, entrapment of the wetting phase due to snap off is not 390 considered in the numerical model. This means that water cannot be trapped as the menisci 391 advance into a pore throat and there is no residual wetting phase left behind.

The misfit between the measured WRC and modelled one may also reflect limitations

393 inherent in the experimental results within this range, where water retention mechanisms other 394 than capillarity may dominate. Several studies on the WRC of soils led us to believe that the 395 water retention characteristic is not only closely related to the pore size distribution but also to 396 the solid pore interfaces (Rossi and Nimmo, 1994; Fayer and Simmons, 1995; Webb, 2000; 397 Tuller and Or, 2005; Khlosi et al. 2006; Silva et al. 2007). The experimental setup used in the 398 laboratory experiment does not provide a highly accurate description of the WRC in the very 399 dry range of the sand. On the other hand, using a wider distribution of the pore throat size gives 400 a much better match with the measured one (Figure 2). This is clearly due to the generation of 401 a few additional thinner pore throats when doubling or tripling the width of the distribution. 402 The penetration of the non-wetting phase into the pore bodies is thus reduced, and more residual 403 water phase is left behind. The characteristics of the pore throat distribution used in the network, 404 such as the mean radius and standard deviation, seem to be a key factor to minimise the 405 difference between experimentally observed WRC and numerically modelled WRC. 406 Optimizing the distribution parameters in this way is similar to optimising fitting parameters in 407 commonly adopted WRC models (Brooks and Corey, 1964; Van Genuchten, 1980). However, 408 the link between modelling parameters and physical properties of the soil is here more explicit. 409 Despite this improvement, the shape of the network, i.e. the number of pores simulated in the 410 horizontal transect on the WRC, remains the principal cause of the misfit between the observed 411 and the modelled WRC. 
413 on the numerical results and to compare them to the experimental results, as we are now able

414 to double or quadruple the number of pores of the network along the cross-section. Such a 415 choice was not possible with a 3D network, as the computational resources do not permit 416 running simulations with a large lattice size. Here, we studied the effect of the shape of the 417 network on the WRC.

418 Simulated water retention curves with networks of $50 \times 50,75 \times 75$, and $100 \times 100$ height by 419 width, but with the same pore throat size (1 sd), are compared with the experimental curve 420 (Figure 3). Numerical results show now a good agreement with the measured curves, especially 421 in the region of pendular water $(S<0.2)$. Compared to the 3D simulation (with a small cross 422 section of $20 \times 20)$, the simulated $(100 \times 100$ based) water retention curve $($ WRC) fits the 423 measured WRC quite well at low water saturation but not at high wetting saturation $(0.2<\mathrm{S}<1)$. 424 This indicates that the larger the horizontal transect is, the higher the trapped water will be. On 425 the other hand, reducing the vertical discretisation of the pore network will lead to an 426 underestimation of the quantity of capillary and funicular water. The behaviour of the pore 427 network is thus shape dependant (function of the number of pores modelled in the cross428 section), as well as size dependant (number of pores modelled along the height). Based on these results, the discrete network model should have enough nodes in all three directions to obtain 430 accurate matching of the experimental WRC.

432 4.2. Modelling of a laboratory experiment on DNAPL/water drainage

433 4.2.1. Displacement behaviour of the water/DNAPL front for a low injection rate

434 The simulations were conducted on a network of $20 \times 20 \times 200$ nodes in the [X,Y,Z] 435 directions. This lattice size was preferred to the $7 \times 7 \times 4858$ lattice size because the modelled 
cross-section is larger, and as shown in section 4.1, the emergent network behaviour is shape dependent.

TheDNAPL arrival times computed at individual pore bodies of a control sectionas defined in the experiment setup (Nsiret al. 2012) have been compared to the measured arrival times for both vertical-upward and vertical-downward flow modes (Figure 4). The 441 corresponding curves were plotted asa function of DNAPLarrival time, normalized by the 442 average arrival time for both the experiments and numerical simulations. As shown in Figure 4, 443 the simulations exhibited behaviours similar to those observed experimentally. In fact, the 444 dimensionless DNAPLarrival timescalculated by the developed modelfor both flow modesare 445 in good agreementwith those obtained in the experiments. The calculated standard deviations 446 are also rather close to the measured values. In fact, the standard deviation values were $14 \mathrm{sec}$ 447 and $75 \mathrm{sec}$, compared to $9 \mathrm{sec}$ and $70 \mathrm{sec}$ measured in the experiments, for the case of vertical448 upward displacement and vertical-downward displacement, respectively. Thus, the model 449 isable to adequately reproduce even the observed displacement instabilities caused by the 450 negative contribution of gravity.

451 In the case of upward displacement, the gravitational component is added to the total 452 potential driving flow and creates an increase in the global inlet pressure. Consequently, the 453 fraction of accessible pores increases and a compact pattern of the invading fluid is established, 454 resulting in a nearly uniform distribution of DNAPL in a porous medium transect. In contrast, 455 because of the negative effect of the gravity forces, the water/DNAPL interface is no longer 456 uniformly distributed over the cross-section in the case of downward displacement and many 457 pore throats become blocked by capillary effects. The number of active invasion paths is 458 therefore lower in this situation, and many vertically oriented fingers may develop in the pore 459 network. 
The final average DNAPLsaturations calculated over the entire network are quite

462 different for upward and downward displacements, confirming that density contrast plays a significant role in the migration of a DNAPL in a porous medium, particularly at low DNAPL injection rates. According to the numerical simulations, we determined an average DNAPL saturation of approximately $72 \%$ in the case of upward displacement but only $41 \%$ for downward displacement. These values are in the same order of magnitude of those obtained in the experiments as an average DNAPL saturation of $66 \%$ and $33 \%$ was obtained for upward and downward displacement, respectively. This difference is mainly due to the stable growth of the water/DNAPL front during upward displacement, whereasthe downward displacement is characterized by flow instabilitiesthat were obviously reproduced quite well with the developed model.

Figure 5 illustrates a 3D representation of the calculated DNAPL saturation along a 1.5$\mathrm{cm}$ length of the equivalent network of the medium sand used in the experiment. The numerical results correspond to an unstable downward displacement. The 3D view of saturation field clearly illustrates the formation of gravity-enhanced fingering in the simulated porous medium. 476 In this condition, only a small amount of displaced fluid (water) comes in contact with the 477 DNAPL and due to the destabilizing effect of gravity, the displacing fluid quickly invades the 478 larger throats and blocks narrow throats where water remains entrapped. Consequently, the 479 advancing DNAPL/water front exhibits several fingers that elongate downwards while 480 advancing through the network.

Based on the DNAPL saturation field computed in the network at a given time, a volume482 averaged DNAPL saturation can be computed over a representative volume of the medium. The 483 resulting macroscopic profiles of volume-averaged DNAPL saturation, as a function of depth 484 for both upward and downward displacement, were then compared to those obtained in the 485 experiment (Figure 6). Similarto the experimental observations, the front behaviourof predicted 
DNAPL saturations appears to be abrupt in the case of upward displacement and presents remarkable fluctuations in the case of downward displacement. The numerical results confirm the experimental observations that the water-DNAPL displacement occurs uniformly when DNAPL is injected from the bottom. However, a heterogeneous cross-section distribution of DNAPL is obtained when the displacing fluid is injected from the top of the column due to both gravity effects and an unfavourable viscosity ratio. The numerical results confirm the experimental findings. However, the calculated DNAPL saturations are higher than the measured values for both displacement modes (upward and downward), especially when a narrow pore throat size distribution is used $(1 \mathrm{sd})$. Significant differences appear, particularly in the region close to the network inlet. For example, at a depth between 10 and $20 \mathrm{~cm}$, differences in saturation may reach $40 \%$ in the case of stable water displacement. Apparently, the simulated number of active and invading fluid paths seems to be relatively higher than the experimental values. Discrepancies to the measurement are reduced and a better fit is obtained, particularly in the case of downward displacement, when a wider pore throat size distribution is used (case where $r_{\max }-r_{\text {mean }}=2 \mathrm{sd}$ ). In this case, much higher capillary pressure in the pore 501 throatis generated and the possibility to overcome it is significantly reduced. This might 502 equilibrate interplay between viscous forces, enhancing the full drainage of water, and capillary 503 forces that are acting to retain displaced fluid. Consequently, the displacement of water by the DNAPL is now better represented by the model leading to simulated DNAPL saturations that are closer to the measured ones.

As previously mentionedwhen modelling WRC (see section 4.1), the relatively higher 507 residual saturations of the invading fluid predicted by the numerical model can also be attributed 508 to the chosen small cross-section of the model. The simulated cross-section of the equivalent 509 network is represented by a small number of nodes $(20 \times 20)$ and remainssmall compared to the 510 reality, which may have limited development of fingering patterns during DNAPL 
displacement. Using a larger section of network may be more appropriate to model the real

512 porous medium, butlarger networks suffer from prohibitive computation times even with 513 powerful computers. To show the effect of the cross-section shape on the emergence of DNAPL 514 displacementbehaviour, we have undertaken a series of additional simulations with a short 515 reduced height (100 nodes)and three sizes of the cross-section, $6 \times 6,12 \times 12$, and $24 \times 24$. Using 516 a much larger cross-sectional size $(>24 \times 24)$ was not manageable with our proper computational 517 resources. Figure 7 shows the DNAPL saturation field and vertical section-averaged DNAPL 518 profile calculated after $8 \mathrm{sec}$ of injection for each of the three cross-sections. The larger the 519 cross-section is, the more advanced the DNAPL fingers are and the less DNAPL saturated the 520 pores are at the inlet section. This clearly underscores the need to increase the cross-section by 521 at least 5 times more nodes in each direction to obtain an improved fit of the experimental 522 results.

\subsubsection{DNAPL pressure at the inlet section}

Although the DNAPL saturation describes the distribution of displacement fluids in the porous medium, it is the pressure build-up that significantly determines the displacement of one

526 fluid by another. Thus, to better understand the interplay between global pressure behaviour 527 and thedisplacement condition (stable or unstable), the variation of the inlet DNAPL pressure 528 as a function of time was predicted by the model and compared to the measurements for the 529 cases of upward displacement and downward displacement, respectively (Figure 8). According 530 to the measured data, the boundary pressure (applied to the outlet section of the experimental 531 system), which was taken into account in the simulation for both upward and downward 532 displacement, corresponded to a water height of $18 \mathrm{~cm}$ and $86 \mathrm{~cm}$, respectively. As observed 533 in the experiments, the pressure increased with time in the case of vertical upward displacement, 534 whereas it decreased in the case of downward displacement. 
Inthe situation where DNAPL is injected at the bottom of the network, the water/DNAPL interface is stabilized by the gravity effect and the potentially destabilizing influence of the viscosity ratio was not significant. The pressure increase is therefore caused by the high density of the invading fluid, which overrides the decrease in viscous pressure drop during the displacement of the less viscous DNAPL. Although the time-dependent increase in DNAPL

540 pressure was well reproduced by the numerical simulations, the calculated pressure increase seems to be slightly lower than that observed in the experiment. This can be explained by the chosen arrangement of throat sizes in the network model. Certain narrow throats that may exist in the real porous medium were not captured in the network and may require a higher displacement pressureto be invaded by the displacing fluid.

In the case of downward DNAPL displacement, the interplay between hydrostatic pressure and viscous pressure changes leads to an overall decrease of the inlet DNAPL pressure. Thesudden increase of pressure illustrated in Figure $8 \mathrm{~b}$ corresponds to the breakthrough of the invading fluid at the outlet section. Here, gravity forces are destabilizing and dominate the movement, resulting in typical DNAPL fingers in the displaced fluid. The number of active 550 invasion paths is therefore lower and the flow section of DNAPL is reduced. The contribution 551 of viscous pressure is also limited and is dominated by the contribution of the higher density of 552 the invading fluid. The combination of all these effects results in decreased inlet pressure. The observed decrease of inlet pressure is correctly reproduced by the numerical model, and the rate

554 at which it decreases seems to be relatively higher than that of the measured one. In addition, 555 the computed breakthrough time is earlier compared to that observed in the laboratory 556 experiment. This can be explained by the number of active invasion paths, which may be lower 557 in the network model than in the real porous medium. The leading fingers as simulated in the model (see Figure 5) are essentially moving straight, pertaining to a zero tortuosity. The 
development of such "no wiggle" fingers decreases the number of pore bodies to invade before

560 reaching the bottom, accelerating the simulated breakthrough.

\subsubsection{Influence of the DNAPL injection rate on gravity fingering}

The DNAPL injection rate is one of the key factors in drainage processes, and it controls

563 the balance between buoyancy, viscous forces, and capillary forces. It has been previously

564 shown (see section 4.2.2) that under a low injection rate, the vertical-downward displacement

565 of water by DNAPL gives rise to several fingers. The invasion of the saturated porous medium

566 is only partial, and the resulting distribution of the DNAPL is thus inhomogeneous. Nsiret al.

567 (2012) showed in previous experimental work that increasing the flow injection rate

568 significantly stabilizes the displacement and may entirely suppress the fingering process during

569 immiscible downward displacement. This scenario was also simulated by the developed model

570 to test its capacity to reproduce such phenomena.

Figure 9shows the comparison of the simulated dimensionless arrival times of the water/DNAPL front with the measured values. The statistical data of both distributions are similar. The calculated standard deviation was very close to the measured value (24 secagainst

$57420 \mathrm{sec}$ measured in the experiment) and much lower than that computed at the low injection rate 575 condition $(75 \mathrm{sec})$. Likewise, the mean arrival time was approximately $489 \mathrm{sec}$, which is 576 compared to the observed time of approximately $512 \mathrm{sec}$. Thus, although gravity fingering is 577 considered to be a highly dynamic process, the numerical results also showthat increasing the 578 flow rate alters its destabilizing effect and leads consequently to the stabilization of the 579 water/DNAPLfront. At a low injection rate, DNAPL moves through a pathway of larger pore 580 bodies and pore throats with the lowest capillary entry pressure because the viscous forces are 581 not significant and do not disrupt the dynamic filling of pore bodies and pore throats. This leads 582 to a ramified invasion and to a preferential path growth in the saturated porous medium. As the 583 flow rate increases, viscous forces become more significant, more meniscibecome mobile, and 
consequently, even fine pore throats may be as overcome as larger ones because of the significant pressure gradient.As a result, a compact pattern of the invading fluid occurs during displacement.

Figure 10 illustrates the macroscopic profile of the volume-averaged DNAPL saturation as a function of depth, calculated when the injection rate of DNAPL was doubled and compared to the measured one. Here, the DNAPL saturations are higher and their spatial distribution is clearly more homogenous compared to those calculated under low injection rate. Such macroscopic behaviouris in good agreement with the experimental observations. However, the simulation results slightly overestimate the monitored DNAPL saturation. Discrepancies are significantly reduced when a wider pore throat size distribution is used in the simulation. The arguments previously detailed in the case of the low injection rate (see section 4.2.2) may also explain the small misfit between measured and calculated average DNAPL saturations. In addition, increasing the size of the network cross-section may improve the fit of the numerical results with the experiment.

\section{Conclusions}

The study presents detailed comparisons between numerical simulations of drainage processes (displacement of water by air, water by DNAPL) and corresponding physical experiments at the scale of a few tens of centimetres. The goodness of fit between the developed pore-scale network model and the experiment seems to be a function of the grid characteristics and modelling domain dimensions.A major feature of the model is its capacity to reasonably well reproducethe redistribution of the fluid phases in the pore space for a given pressure change, a modification of the applied displacement condition (stable/unstable) or for a rate injection increase. In fact, the predicted water retention curve matched well globally with that obtained in the experiment, especially within the region of higherwater saturation $(0.2<\mathrm{S}<1)$. 
The predicted distribution of arrival times of the water/DNAPL front computed at a given cross-

610 section are in good agreement with the experimental data obtained for a given displacement 611 scenario. The slightly higher DNAPL saturations predicted by the numerical model are

612 essentially attributed to the chosen small cross-section of the equivalent network. In the actual 613 version of the model, the lattice sizes are limited by the computation time and space memory 614 and remain too far to adequately well representthe whole cross-section of the real porous 615 medium. Thus, a network model with enough nodesfor the cross-section allowsdistinct 616 behaviourof immiscible displacement, such as DNAPL gravity-driving fingering to emerge 617 sufficiently from the interactions of the various pores and throats that constitute the network 618 and the various forces taken in account in the model. This may reduce the differences between 619 experimentally determined saturations and modelled saturations. In this context, further 620 developments of the numerical code, such as implementation of an iterative and more powerful 621 solver, should be undertaken to reduce and optimize the CPU time. Complementary studies are 622 also necessary to test additional features of the pore-space geometry of the porous medium. The 623 model can also be extended to simulate the imbibition process and promote application of the 624 model to reproduce the experimentally obtained residual DNAPL saturations.

625

\section{Acknowledgements}

Financial support for this research was received from the French Agency for Research

628 (AgenceNationale de la Recherche), through the Labex Voltaire ANR-10-LABX-100-01. The 629 authors benefited also from the use of the cluster at the Centre de CalculScientifiqueen region 630 Centre (CCSC) in France. Laurent Catherine is acknowledged for his technical assistance. 631 Furthermore, theauthorswouldlike to thanktheRegionAlsace, the GDR «Hydrodynamique et 632 Transfertsdans les HydrosystèmesSouterrains » (INSU-CNRS), and theConseilScientifique de 633 l'Université de Strasbourgfortheirfinancialsupport. 


\section{References}

636

Aker, E., Maloy, K.J., Hansen, A., Bartouni, G., 1998. A two-dimensional network simulator for two-phase flow in porous media. Transp. Porous Med. 32, 163-186

Aggelopoulos, C.A., Tsakiroglou, C.D., 2009. A multi-flow path model for the interpretation of immiscible displacement experiments in heterogeneous soil columns. J. Contam. Hydrol. 105, 146-160

Alexandra, R., Gerhard, J.I., Kueper, B.H., 2012. Hydraulic displacement of dense nonaqueous phase liquids for source zone stabilization. Ground Water 50, 765-774

Al-Raoush, R.I., Willson, C.S., 2005. A pore-scale investigation of a multiphase porous media system. J. Contam. Hydrol. 77, 67-89

Bettahar, B., Ducreux, J., Schäfer, G., Van Dorpe, V., 1999. Surfactant Enhanced In Situ Remediation of LNAPL Contaminated Aquifers: Large Scale Studies on a Controlled Experimental Site. Transp. Porous Med. 37, 276-186

Birovljev, A., Furuberg, L., Feder, J., Jøssang, T., Måløy, K.J., Aharony, A., 1991. Gravity invasion percolation in 2 dimensions-experiment and simulation. Phys. Rev. Lett. 67, 584-587

Bohy, M., Dridi, L., Schäfer, G., Razakarisoa, O., 2006. Transport of a mixture of chlorinated solvents vapors in the vadose zone of a sandy aquifer: Experimental study and numerical modeling. Vadose Zone J. 5, 539-553

Brailovsky, I., Babchin, A., Frankel, M., Sivashinsky, G., 2006. Fingering instability in water-oil displacement. Transp. Porous Med. 63, 363-380

Brooks, R., Corey, A., 1964. Hydraulic Properties of Porous Media, p. 3. Colorado State 
658 Bultreys, T., Van Hoorebeke, L., Cnudde, V., 2015. Multi-scale, micro-computed tomography- based pore network models to simulate drainage in heterogeneous rocks. Adv. Water Resour.78, 36-49

Cheng Z., Gao B., Xu H., Sun Y., Shi X., Wu J., 2016. Effects of surface active agents on DNAPL migration and distribution in saturated porous media. Sci Total Environ. 571,

Cotel, S., 2008. Étude des transferts sol/nappe/atmosphère/bâtiments : Application aux sols 665 pollués par des composés organiques volatils. Thèse de doctorat. Université Joseph Fourier, Grenoble, France, 203p

Dodds, J.A., 1980. The porosity and contact points in multicomponent random sphere packings calculated by a simple statistical geometric model. J. Colloid and Interface Sci. $77,317-327$

Dong, H., Blunt, M.J., 2009. Pore-network extraction from micro-computerized-tomography images. Phys. Rev. E. doi:10.1103/PhysRevE.80.036307

Dong, B., Yan, Y., Li, W.Z., 2011. LBM simulation of viscous fingering phenomenon in immiscible displacement of two fluids in porous media. Transp. Porous Med. 88, 293-

Erning, K., Grandel, S., Dahmke, A., Schäfer, D., 2012. Simulation of DNAPL infiltration 314 and spreading behaviour in the saturated zone at varying flow velocities and alternating subsurface geometries. Environ. Earth Sci. 65, 1119-1131 the subsurface: evolution of knowledge and understanding. Water Resour. Res. 51, 4861-4874 
681 Ewing, R.P., Berkowitz, B., 1998. A generalized growth model for simulating initial migration of dense non-aqueous phase liquids. Water Resour. Res. 34, 611-622

683

684

685

686

687

688

689

690

691

692

693

694

695

696

697

698

699

Fayer, M.J., and Simmons, C.S., 1995. Modified soil water retention functions for all matric suctions. Water Resour. Res. 31, 1233-1238

Fayers, F.J., Zhou, D., 1996. On the importance of gravity and three-phase flow in gas displacement processesJ. Petroleum Science and Engineering. 15, 321-341

Ferer, M., Bromhal, G.S., Smith, D.H., 2007. Crossover from capillary fingering to compact invasion for two-phase drainage with stable viscosity ratios. Adv. Water Resour. 30, 284-299

Gao, S., Meegoda, J.N., Hu, L., 2012. Two methods for pore network of porous media. Int. J. Numer. Anal. Methods Geomech. 36, 1954-1970

Glass, R.J., Conrad, S.H., Yarrington, L., 2001. Gravity destabilized non-wetting phase invasion in macroheterogeneous porous media: Near pore scale macro modified invasion percolation model, Water Resour. Res. 37, 1197-1207

Helge, K., Michael, D.A., Celia, A., 1999. A dynamic network model for two-phase immiscible flow. Comput. Geosci. 3, 1-22

Hao, L., Cheng, P., 2010. Pore-scale simulations on relative permeabilities of porous media by lattice Boltzmann method. Int. J. Heat Mass Transf. 53, 1908-1913

Jia, C., Shing, K., Yortsos,Y.C., 1999. Visualization and simulation of non-aqueous phase liquids solubilization in pore networks. J. Contam. Hydrol.35, 363-387

Jivkov, A.P., Hollis, C., Etiese, F., McDonald, S.A., Withers, P.J., 2013. A novel architecture for pore network modelling with applications to permeability of porous media. J. Hydrol. $486,246-258$ 
Joekar-Niasar, V., Hassanizadeh, S. M., 2012. Analysis of fundamentals of two-phase flow in porous media using dynamic pore-network models: A review. Environ. Sci. Technol. 42, 1895-1976

Joekar-Niasar, V., Hassanizadeh, S.M., Pyrak-Nolte, L.J., Berentsen, C., 2009. Simulating drainage and imbibition experiments in a high-porosity micromodel using an unstructured pore network model. Water Resour. Res., doi: 10.1029/2007WR006641

Kallel, W., van Dijke, M.J., Sorbie, K.S., Wood, R., 2017. Pore-scale modeling of wettability alteration during primary drainage. Water Resour. Res. 53, 1891-1907

Khataniar, S., Peters, E.J., 1992. The effect of reservoir heterogeneity on the performance of unstable displacements. J. Pet. Sci. Eng. 7, 263-281

Khlosi, M., Cornelis, W.M., Gabriels, D., Sin, G., 2006. Simple modification to describe the soil water retention curve between saturation and oven dryness. Water Resour. Res., doi: 10.1029/2005WR004699

Kiriakidis, D., Mitsoulis, G.E., Neale, G.H., 1991. Linear displacement of a wetting fluid by an immiscible non-wetting fluid in a porous medium: a predictive algorithm. Can. J. Chem. Eng. 69, 557-563

Kokkinaki A., O’Carroll D. M., Werth C. J., Sleep B.E., 2013. Coupled simulation of DNAPL infiltration and dissolution in three-dimensional heterogeneous domains: Process model validation. Water Resour. Res. 49, 7023-7036

Koplik, J., Lasseter, T.J., 1985. Two phase flow in random network models of porous media. SPE 30, 89-100

Kueper, B.H., Frind, E.O., 1989. An overview of immiscible fingering in porous media. J. Contam. Hydrol. 2, 95-110 
Laroche, F., Vizika, O., 2005. Two-phase flow properties prediction from small-scale data using pore-network modeling. Transp. Porous Med. 6, 77-91

Lenormand, R, Touboul, E., Zarcone, C., 1998. Numerical models and experiments on immiscible displacements in porous media. J. Fluid Mech. 189, 165-187

Liu, H., Valocchi, A.J., Werth, C., Kang, Q., Oostrom, M., 2014. Pore-scale simulation of liquid $\mathrm{CO}_{2}$ displacement of water using a two-phase lattice Boltzmann model. Adv. Water Resour. 73, 144-158

Liu, H., Zhang, Y., Valocchi, A.J., 2015. Lattice Boltzmann simulation of immiscible fluid displacement in porous media: Homogeneous versus heterogeneous pore network. Phys. Fluids, doi: 10.1063/1.4921611

Liu, H., Valocchi, A.J, Kang, Q., Werth C., 2013. Pore-scale simulations of gas displacing liquid in a homogeneous pore network using the lattice Boltzmann method. Transp. Porous Med. 99, 555-580

Nayagum, D., Schäfer, G., Mose, R., 2004. Modeling two-phase incompressible flow in porous media using mixed hybrid and discontinuous finite elements. Comput. Geosci. 8, $49-73$

Nordhaug, H.F., Celia, M., Dahle, H.K., 2003. A pore network model for calculation of interfacial velocities. Adv. Water Resour. 26, 1061-1074

Nsir, K., Schäfer, G., 2010. A pore-throat-model based on grain size distribution to quantify gravity-dominated DNAPL instabilities in water saturated homogeneous porous medium. C.R. Geosci. 342, 881-891

Nsir, K., Schäfer, G., di Chiara R., Razakarisoa O., Toussaint R., 2012. Laboratory experiments on DNAPL gravity fingering in water-saturated. Int.J. Multiph. Flow 40, $83-92$ 
Prodanovic, M., Lindquist, W., Seright, R.S., 2007. 3D image-based characterization of fluid displacement in a Berea core. Water Resour. Res. 30, 214-226

Raoof, A., Hassanizadeh, S.M., 2010. A new method for generating pore network models of porous media. Transp. Porous Med. 81, 391-407

Raoof, A., Hassanizadeh, S.M., Leijnse, A., 2010. Upscaling transport of adsorbing solutes in porous media: pore-network modeling.Vadose Zone Journal 9, 624-636.

Riaz, A., Tchelepi, H.A., 2006. Influence of Relative Permeability on the Stability Characteristics of Immiscible Flow in Porous Media. Transp. Porous Med. 64, 315-338

Rouault, Y., Assouline, S., 1998. A probabilistic approach towards modelling the relationships between particle and pore size distributions: the multicomponent packed sphere case. Powder Technol. 96, 33-41

Rossi, C., Nimmo, J.R., 1994. Modeling of soil water retention from saturation to oven dryness. Water Resour. Res. 30, 701-708

Schneider, L., di Chiara Roupert, R., Schäfer, G., Helluy, P., 2015. Highly gravity-driven flow of an NAPL in water-saturated porous media using Discontinuous Galerkin FiniteElement Method with a generalised Godunov scheme. ComputGeosci 19, 855-876.

Sheng, Q., Thompson, K., 2013. Dynamic coupling of pore-scale and reservoir-scale models for multiphase flow, Water Resour. Res. 49, 5973-5988

Silva, O., Grifoll, J., 2007. Non-passive transport of volatile organic compounds in the unsaturated zone. Adv. Water. Resour. 30, 794-807

Sleep, B.E., Beranger, S., Reinecke, S., Filion, Y., 2015. DNAPL accumulation in well sand DNAPL recovery from wells: Model development and application to a laboratory study. Adv. Water Resour. 85, 109-119 
Singh, M., Mohenty, K.K., 2003. Dynamic network for drainage through three dimensional porous materials. Chem. Eng. Sci. 58, 1-18

Tuller, M., Or, D., 2005. Water films and scaling of soil characteristic curves at low water contents. Water Resour. Res., doi: 10.1029/2005WR004142

Tørå, G., Øren, P.E, Hansen, A., 2012. A dynamic network model for two-phase flow in porous media. Transp. Porous Med. 92, 145-164

Van Genuchten, M.T., 1980. A closed-form equation for predicting the hydraulic conductivity of unsaturated soils. Soil Sci. Soc. Am. J. 44, 892-898

Washburn, E.W., 1921. The dynamics of capillary flow, Phys. Rev. 17, 273-283 (1921)

Webb, S.W., 2000. A simple extension of two-phase characteristic curves to include the dry region. Water Resour. Res. 36, 1425-1430

Wu, M., Cheng, Z., Wu, J., Wu, J., 2017. Estimation of representative elementary volume for DNAPL saturation and DNAPL-water interfacial areas in 2D heterogeneous porous media. J. Hydrol. 549, 12-26

Xiong, Q., Baychev, T.G., Jivkov, A.P., 2016. Review of pore network modelling of porous media: Experimental characterisations, network constructions and applications to reactive transport. J. Contam. Hydrol.192, 101-117

Zhang, Z.F., Smith, J.E., 2001. The velocity of DNAPL fingering in water-saturated porous media: laboratory experiments and a mobile-immobile-zone model. J. Contam. Hydrol. $49,335-353$

Zhang, L, Kang, Q., Yao, J., Gao, Y., Sun, Z., Liu, H., Valocchi A.J., 2015. Pore scale simulation of liquid and gas two phase flow based on digital core technology. Science China Technological Sciences. 58, 1375-1384 
Table 1: Minimum, mean, and maximum radii of pore bodies and pore throats derived from the sphere-packing model.

\begin{tabular}{|c|c|c|c|c|}
\hline \multirow{3}{*}{ Pore-body radius [mm] } & $\operatorname{minimum}\left(r_{\min }\right)$ & \multicolumn{3}{|c|}{0.042} \\
\hline & mean $\left(r_{\text {mean }}\right)$ & \multicolumn{3}{|c|}{0.048} \\
\hline & $\operatorname{maximum}\left(\mathrm{r}_{\max }\right)$ & \multicolumn{3}{|c|}{0.054} \\
\hline \multirow{4}{*}{ Pore-throat radius [mm] } & & $r_{\text {max }}-r_{\text {mean }}=s$ & $r_{\text {max }}-r_{\text {mean }}=2 \mathrm{sd}$ & $r_{\max }-r_{\text {mean }}=3 \mathrm{sd}$ \\
\hline & $\operatorname{minimum}\left(\mathrm{r}_{\min }\right)$ & 0.026 & 0.022 & 0.018 \\
\hline & mean ( $\left.r_{\text {mean }}\right)$ & 0.030 & 0.030 & 0.030 \\
\hline & $\operatorname{maximum}\left(r_{\max }\right)$ & 0.034 & 0.038 & 0.042 \\
\hline
\end{tabular}

799

800

$801 \quad$ Figure captions

802 Fig. 1: Flow chart of the pore-scale network model. The time-stepping process is repeated until

803 a stopping condition is (dynamically) reached, or when equilibrium state is achieved, i.e., all

804 the interfaces are blocked.

805 Fig. 2: Experimental and modelled WRC of the quartz sand used in the laboratory experiments.

806 Simulations with a 3D lattice network $(20 \times 20 \times 200)$ and 3 different pore throat radii

807 distributions. The capillary pressure value is expressed as equivalent water head $\left(\mathrm{m} \mathrm{H}_{2} \mathrm{O}\right)$.

808 Fig. 3: Experimental and modelled WRC of the quartz sand used in the laboratory experiments.

809 Simulations with a 2D lattice network with 3 different sizes $(50 \times 50,75 \times 75$, and $100 \times 100)$ and

810 a given pore-throat radii distribution $\left(\mathrm{r}_{\max }-\mathrm{r}_{\operatorname{mean}}=1 \mathrm{sd}\right)$. The capillary pressure value is expressed

811 as equivalent water head $\left(\mathrm{m} \mathrm{H}_{2} \mathrm{O}\right)$.

812 Fig. 4: Measured and simulated dimensionless arrival times of the water/DNAPL front using a

813 3D lattice network $(7 \times 7 \times 4858)$ in both vertical-upward flow mode and downward flow mode:

814 The case of drainage of water-saturated medium sand at low DNAPL injection flow rate $815\left(\mathrm{Q}=29.6 \times 10^{-4} \mathrm{~mL} / \mathrm{min}\right) . \mathrm{t}_{\text {mean }}$ is the average arrival time of the DNAPL front.

816 Fig. 5: The 3D view of the simulated DNAPL saturation field for vertical-downward drainage 817 of saturated medium-sized sand at low DNAPL injection flow rate using a 3D lattice network 
$818(7 \times 7 \times 4858$ nodes): (a) near the inlet, between $\mathrm{z}=0 \mathrm{~cm}$ and $\mathrm{z}=-1.5 \mathrm{~cm}$; (b) in the middle of the

819 soil column, between $\mathrm{z}=-34 \mathrm{~cm}$ and $\mathrm{z}=-35.5 \mathrm{~cm}$; and (c) near the outlet, between $\mathrm{z}=-66.5 \mathrm{~cm}$ 820 and $\mathrm{z}=-68 \mathrm{~cm}$.

821 Fig. 6: Measured and simulated saturation as a function of depth at the end of the experiment 822 using a 3D lattice network $(20 \times 20 \times 200)$ with 3 different pore throat radius distributions: (a) in 823 the case of vertical-upward drainage of water-saturated medium sand at low DNAPL injection 824 flow rate and (b)in the case of vertical-downward drainage of water-saturated medium sand at 825 low DNAPL injection flow rate.

826 Fig. 7: Effect of the size of the cross-section $(6 \times 6,12 \times 12$, and $24 \times 24)$ on the simulated DNAPL 827 saturation field and cross-section averaged DNAPL saturation profile: in the case of vertical828 downward drainage of saturated medium-sized sand at low DNAPL injection flow rate $(\mathrm{Q}=$ $\left.82929.6 \times 10^{-4} \mathrm{~mL} / \mathrm{min}\right)$, after $8 \mathrm{sec}$ of DNAPL injection.

830 Fig. 8: Measured and simulated inlet DNAPL pressure as a function of time using a 3D network

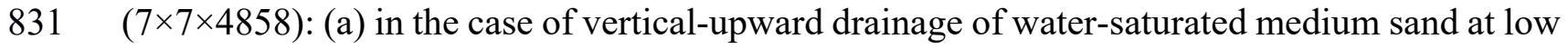
832 DNAPL injection flow rate and (b) in the case of vertical-downward drainage of water-saturated 833 medium sand at low DNAPL injection flow rate $\left(\mathrm{Q}=29.6 \times 10^{-4} \mathrm{~mL} / \mathrm{min}\right)$.

834 Fig. 9: Measured and simulated dimensionless arrival times of the water/DNAPL front in 835 medium sand.The case of vertical-downward drainage of water-saturated medium sand at high 836 DNAPL injection flow rate $\left(\mathrm{Q}=59.2 \times 10^{-4} \mathrm{~mL} / \mathrm{min}\right) . t_{\text {mean }}$ is the average arrival time of the 837 DNAPL front

838 Fig. 10: Measured and predicted DNAPL saturation as a function of depth at the end of the 839 experiment using a 3D lattice network $(20 \times 20 \times 200)$ with 3 different pore throat radius 840 distributions. The case of vertical-downward drainage of water-saturated medium sand at high 841 DNAPL injection flow rate $\left(\mathrm{Q}=59.2 \times 10^{-4} \mathrm{~mL} / \mathrm{min}\right)$. 
